\title{
Bu Shen Yang Xue Prescription Has Treating Effect on Endometrial Cancer through FSH/PI3K/AKT/Gankyrin/HIF- $\alpha /$ cyclinD 1 Pathway in Ishikawa Cells
}

\author{
Yue-qun Chen, ${ }^{1}$ Hua-li Fei, ${ }^{2}$ and Hong-li Zhu $\mathbb{D}^{1}$ \\ ${ }^{1}$ Department of Obstetrics and Gynecology, Affiliated Hangzhou First People’s Hospital, \\ Zhejiang University School of Medicine, Hangzhou 310006, Zhejiang Province, China \\ ${ }^{2}$ Department of Obstetrics and Gynecology, Hangzhou Women's Hospital, Hangzhou 310008, Zhejiang Province, China
}

Correspondence should be addressed to Hong-li Zhu; cyqziyan@163.com

Received 16 July 2018; Revised 2 September 2018; Accepted 26 September 2018; Published 9 October 2018

Academic Editor: Caigan Du

Copyright (C) 2018 Yue-qun Chen et al. This is an open access article distributed under the Creative Commons Attribution License, which permits unrestricted use, distribution, and reproduction in any medium, provided the original work is properly cited.

Background. The formulation of Bu Shen Yang Xue (BSYX) has been clinically used in treating gynecologic disease in China, especially for the development of the endometrium. Endometrial carcinoma is the most common malignant tumor of the female genital tract in developed countries. And few studies have been reported on the antitumor activity of BSYX. Therefore, this study aimed to investigate the effect of BSYX on endometrial cancer and make an initial discussion of the underlining mechanisms in Ishikawa cells. Methods and Results. Firstly, $60 \mathrm{SPF}$ female nude mice were randomly divided into control group, model group, BSYX group, and positive group. The models of subcutaneous tumor xenograft of nude mice were established by injection of human endometrial carcinoma cell line Ishikawa tumor cell suspension. Compared with model group, BSYX reduced effectively tumor volume and changed pathological feature in mice tumor issue. Meanwhile, proteins from tumor issues were detected by western blot analysis. The protein levels of follicle-stimulating hormone receptor (FSHR), p-Akt/Akt, Gankyrin, and cyclinD1 in the model group were higher than those in control group but the expression in BSYX group was lower than that in the model group. The hypoxia inducible factor alpha (HIF- $\alpha$ ) protein level in the model group was lower than those in control group and upregulated in BSYX group. In addition, Ishikawa cells were cultured and then exposed to follicle-stimulating hormone (FSH), LY294002, a highly selective PI3K inhibitor and serum containing BSYX, respectively. LY294002 and BSYX markedly decreased the cancer cell viability and migration ability and increased the apoptosis rate. FSH promoted the cancer cell ability and migration ability. LY294002 and BSYX evidently downregulated the proteins levels of FSHR, p-Akt/Akt, Gankyrin, and cyclinD1 and upregulated the expression of HIF- $\alpha$ protein, and FSH was on the opposite. Conclusions. Taken together, our results showed that the formulation of BSYX had antitumor effect on endometrial cancer in vivo and in vitro and was related with FSH/PI3K/AKT/Gankyrin/HIF- $\alpha /$ cyclinD1 transduction pathway.

\section{Introduction}

Endometrial cancer, also known as uterine body cancer, refers to a group of epithelial malignant tumors that originated from the endometrium, most of which originated from the endometrial gland and it is called endometrial adenocarcinoma. Endometrial cancer is one of the three most common malignant tumors in the female reproductive tract, accounting for $8 \%$ of the total malignant tumors in the female body and $20 \%$ to $30 \%$ of the malignant tumors in the female reproductive tract [1]. In recent years, the incidence of endometrial cancer has increased significantly at home and abroad due to the prolongation of human life and the widespread use of exogenous estrogen, and the age of onset tends to be younger [2,3]. Several risk factors have been reported to be involved in endometrial carcinoma, such as hypertension, postmenopausal status, infertility, family history, and long-term use of estrogens. In spite of 
tremendous advances made in the diagnosis and treatment of endometrial cancer in recent years, the treatment of advanced stages of the disease is still difficult because of the unclear pathological mechanisms. Early-stage endometrial cancer has a high survival rate, while approximately $30 \%$ of patients are diagnosed with advanced stage disease [4]. The treatment principle of endometrium is mainly surgery, supplemented by radiotherapy, chemotherapy, endocrine therapy, and biological treatment. Procedure is capable of tumor resection, but there are residual cancer tumor emboli in regional lymph node metastasis, vascular, radiotherapy, chemotherapy, and hematopoietic system leading to certain side effects on the digestive tract, and the prognosis is not ideal. Therefore, it is very important to develop treatment strategies for this disease.

Treatment with traditional Chinese medicine (TCM) can improve the effect on radiotherapy and chemotherapy in clinic and also reduce the toxic and side effects of radiotherapy and chemotherapy on digestive tract and hematopoietic system. For patients with advanced endometrial cancer or those who cannot operate, radiotherapy and chemotherapy can also be treated with TCM. TCM holds that the main pathogenic factors of endometrial cancer are related to Yin and Yang deficiency, fatigue, faint praise, dampness, and heat. $\mathrm{Bu}$ shen Yang xue (BSYX) prescription was derived from the works of traditional Chinese medicine named Jing yue's complete work and was made up of many herbs: Herba epimedium (Yin yang huo), dodder (Tu si zi), eucommia ulmoides (Du zhong), dogwood (Shan zhu yu), ripe radix rehmanniae (Shu di huang), salvia miltiorrhiza (Dang shen), wolfberry (Gou qi), angelica sinensis (Dang gui), paeoniae (Shao yao), and Ligusticum wallichii (chuan xiong), mixed with licorice (Gan cao) and herbs. BSYX has the characteristics of nourishing liver and kidney, invigorating qi and activating blood to collect Yin, replenishing without stagnation, nourishing without satiety and satiety, and making kidney gas abundant and decayed to heaven. BSYX has been used in treating gynecologic disease in clinic, including immune premature ovarian failure, infertility, and acted on the development of the endometrium [5-7]. So in this study, we investigated whether BSYX prescription was effective in the therapy of endometrial cancer or not.

Endometrial carcinoma is a hormone-dependent malignancy. When estrogen decreases after menopause, facilitates follicle-stimulating hormone (FSH) will increase. FSH is the core hormone in mammalian reproductive process. High FSH has large impact on women's reproductive and perimenopausal physiological pathology, and the signaling pathway downstream of FSH receptor (FSHR) has become a new research field. During human proliferation and secretion, FSHR protein is expressed in endometrial epithelial cells and interstitial cells, and the expression of secretory phase is increased. FSH may regulate endometrial function [8].

There is evidence that the phosphoinositide-3-kinase (PI3K) pathway is the most commonly activated pathway in cancer, such as human cancer cell lines [9]. More importantly, PI3K signal transduction pathway was frequently altered within endometrial carcinoma, and the activation of
PI3K/AKT pathway could increase the incidence of endometrial carcinoma and lead to poorer prognosis [10, 11]. In addition, Gankyrin is a member of the family of anchor protein repeat sequences and signaling pathways can be regulated to mediate the development of cancer by the interaction of proteins with proteins [12]. Gankyrin plays an essential role in estrogen-driven and G-protein coupled receptor 30mediated endometrial carcinoma cell proliferation via the PTEN/PI3K/AKT signaling pathway [13]. Studies showed that, after FSH binds to FSHR in ovarian cancer, PI3K/AKT pathway was activated, and the downstream gene Gankyrin expression would increase correspondingly. The expression of Gankyrin mediates the ubiquitination of hypoxia inducible factor- $1 \alpha$ (HIF- $1 \alpha)$ and inhibits the nucleation of HIF- $1 \alpha$, thereby reducing the binding with cyclin D1 promoter, and the expression of cyclin D1 increases correspondingly. On the other hand, Gankyrin expression inhibits PTEN expression and promotes cell proliferation [14]. Given those factors, we conducted this present study about BSYX performed antitumor effect on endometrial cancer and the relationship of FSH/ PI3K /AKT/Gankyrin/HIF- $\alpha$ /cyclinD 1 pathway in vivo and in vitro.

\section{Materials and Methods}

2.1. Preparation of BSYX Decoction. BSYX prescription contains eleven medicinal materials that include Herba epimedium, dodder, eucommia ulmoides, dogwood, ripe radix rehmanniae, salvia miltiorrhiza, wolfberry, angelica sinensis, paeoniae, and Ligusticum wallichii, mixed with licorice. These dried slices were acquired from Beijing Tongrentang Drugstore in China. The raw herbs were first boiled by 8 -fold of water $(\mathrm{v} / \mathrm{w})$ for $2 \mathrm{~h}$ at atmosphere pressure. The decoction was then collected; an additional 6-fold of water was further added and boiled for $1.5 \mathrm{~h}$. The second decoction was separated and mixed with the first, which was then concentrated by heating at low-atmosphere pressure condition. Finally an extract of $1 \mathrm{mg} / \mathrm{mL}$ was prepared in a large scare for detailed experimental investigations.

2.2. Cell Culture. Human endometrial carcinoma Ishikawa cells, an estrogen-responsive cell line derived from a welldifferentiated endometrioid carcinoma, were obtained from the National Infrastructure of Cell Resource, China. The cells were maintained in Dulbecco's modified Eagle's medium (DMEM high glucose) (Gibco BRL. Grand Island, NY, USA), with $10 \%$ fetal bovine serum (Gibco BRL. Grand Island, NY, USA), $100 \mathrm{U} / \mathrm{ml}$ penicillin, and $100 \mathrm{U} / \mathrm{mL}$ of streptomycin in a humidified atmosphere of $5 \% \mathrm{CO}_{2}$ at $37^{\circ} \mathrm{C}$.

2.3. Animal Experiments. 60 SPF four-week-old female nude mice $(B A L B / C)$ were provided by zhejiang Chinese Medical University Laboratory Animal Research Center. Mice were randomly divided into control group, model group, BSYX group $(2.5,5$ and $10 \mathrm{~g} / \mathrm{kg})$, and Cisplatin group, and each group had 10 mice. Besides the control group, the models of subcutaneous tumor xenograft of nude mice with human endometrial carcinoma were established by right 
scapular subcutaneous injection of $1 \times 10^{7}$ Ishikawa tumor cell suspension. Tumor formation was about three weeks. Then BSYX group was treated with intragastric injection administration of BSYX, and the control group and model group were injected with normal saline for 14 days. Tumor volumes were gauged with vernier calipers regularly and the growth curves for tumors were plotted as mean \pm SD of tumors from 8 mice. Tumor volume was evaluated according to the following formula: $1 / 2 \times \mathrm{L}^{2} \times \mathrm{W}(\mathrm{L}=$ tumor length and $\mathrm{W}=$ tumor width $)$. Antitumor rate $=(\mathrm{C}-\mathrm{T}) / \mathrm{C} \times 100 \%(\mathrm{C}=$ the average volume of the tumor in the model group, $\mathrm{T}=$ the average volume of the transplanted tumor in the treatment group).

The mice were housed in a specific pathogen-free environment suitable for survival and were observed and measured on a regular basis. Later the mice were sacrificed and the tumors excised to compare the size of the tumor. All mouse experiments were approved by the animal ethics committee of Zhejiang University.

2.4. Hematoxylin-Eosin (HE) Staining. The tumor tissues were immersion-fixed in $10 \%$ neutral buffered formaldehyde solution and embedded in paraffin for a light microscopic study. The sections of formalin-fixed, paraffin-embedded tissue samples were deparaffinized in xylene and rehydrated in graded ethanols. The specimens were routinely processed and stained with HE for histopathological classification.

2.5. The Serum Containing BSYX Preparation. The ICR female mice weighing $18-22 \mathrm{~g}$ were randomly divided into following groups $(\mathrm{n}=10)$ : a control group (normal saline); three BSYX groups $(2.5 \mathrm{~g} / \mathrm{kg}, 5 \mathrm{~g} / \mathrm{kg}$, and $10 \mathrm{~g} / \mathrm{kg})$. The control saline or BSYX groups were administered via intragastric injection every 2 days for five times. At day 14, all the animals were sacrificed and the drug serum and the blank serum were separated from heart blood. The blood was centrifuged at $4000 \mathrm{rpm}$ for $10 \mathrm{~min}$ at $4^{\circ} \mathrm{C}$ to obtain serum. The serum was labeled and stored at $-70^{\circ} \mathrm{C}$.

2.6. Cell Treatment and Grouping. Ishikawa cells were cultured and then divided into six groups: normal control group, FSH $(50 \mathrm{mIU} / \mathrm{ml}), \mathrm{LY} 294002(15 \mu \mathrm{mol} / \mathrm{L})$, blank serum, and drug serum from mice treated BSYX $(2.5,5$, and $10 \mathrm{mg} / \mathrm{kg})$. The concentrations of FSH and LY294002 were selected by reference to relevant literatures about endometrial cancer cells. $[15,16]$

2.7. CCK8 Assay. The CCK8 assay was performed using a CCK8 kit following the manufacturer's protocol (Dojindo, Kumamoto, Japan). Briefly, cells were plated into 96-well plates $\left(2 \times 10^{3}\right.$ cells per well) in $100 \mu \mathrm{L}$ of culture medium and incubated for $24 \mathrm{~h}$ at $37^{\circ} \mathrm{C}$. CCK- 8 solution $(100 \mu \mathrm{L} /$ well $)$ was added for another $2 \mathrm{~h}$ and then incubated for $24 \mathrm{~h}$. Before reading the plate, it is important to mix gently on an orbital shaker for 1 minute to ensure homogeneous distribution of color. Then, the absorbance was measured at $450 \mathrm{~nm}$ with a Thermomax microplate reader (Molecular Devices,
Sunnyvale, CA, USA). Each experiment was repeated three times.

2.8. Annexin V-FITC/PI Staining. Cell apoptosis/necrosis was evaluated using Annexin V-FITC/ PI staining. Cells were plated into 6-well plates. After treatment for $24 \mathrm{~h}$, cells were collected and washed twice with cold phosphate-buffered saline (PBS). The cells were then resuspended in $500 \mu \mathrm{L}$ buffer with $100 \mu \mathrm{L}$ of buffer and $5 \mu \mathrm{L}$ Annexin V- FITC and $5 \mu \mathrm{L}$ propidium iodide (PI) and incubated for $15 \mathrm{~min}$ in the dark at room temperature. Binding buffer $(250 \mu \mathrm{L})$ was added to each tube, and the apoptosis rate was determined by flow cytometry within $1 \mathrm{~h}$. Annexin V-/PI-cells are viable, annexin $\mathrm{V}+$ /PI- cells are in early apoptosis, and annexin $\mathrm{V}+/ \mathrm{PI}+$ cells are necrotic or in late apoptosis

2.9. Cell Migration Rate Detection. Cells were seeded in 6well plates at the density of $5 \times 10^{5}$ cells and cultured until confluence. The cell monolayer was scratched with a sterile pipette yellow tip to produce a straight line, and the debris was washed out with culture medium. Medium and substances for treatment were renovated at $24 \mathrm{~h}$ or $48 \mathrm{~h}$. The open gap was photographed with a camera at phase contrast microscope at the indicated times. The rate of healing was estimated by ImageJ software based on the area (in which the length is predetermined and the wideness varies) free of cells. Each determination represents the average of 3 individual experiments.

2.10. Western Blot. The total proteins from cell or tumor issue were harvested and lysed in RIPA buffer containing $1 \mathrm{mM}$ phenyl methane sul-fonyl fluoride (Beyotime Biotechnology, Shanghai, China) and mixed with loading buffer. Protein concentration was determined by BCA kit (Beyotime

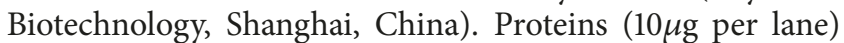
were separated by sodium dodecylsulfate polyacrylamide gel electrophoresis and then transferred to a polyvinylidene difluoride membrane (PVDF, Millipore, Billerica, MA, USA). The membranes were blocked with PBS containing $5 \%$ skim milk for $2 \mathrm{~h}$ and then incubated overnight with primary antibodies against Bax (diluted at 1:1000; \#5023, CST), Bcl-2 (diluted at 1:1000; \#4223, CST) AKT (diluted at 1:1000; \#4691, CST), phospho (p)-AKT (diluted at 1:1000; \#4060, CST), FSHR (diluted at 1:500; ab75200, abcam), Gankyrin (diluted at1:500;\#12985, CST), HIF- $\alpha$ (diluted at 1:500; ab179483, abcam), cyclin D1(diluted at 1:500;\#2978, CST), and GAPDH (diluted at 1:1000; \#5174, CST) at $4^{\circ} \mathrm{C}$. The membranes were washed three times with TBST (Trisbuffered saline containing Tween-20) and then anti-rabbit secondary antibodies (1:5000) were added. Following $2 \mathrm{~h}$ incubation at room temperature, the protein bands were visualized by enhanced chemiluminescence (ECL, Beyotime Biotechnology, Shanghai, China) according to the manufacturer's instruction. The images were obtained using MiniPROTEAN gel imaging system. GAPDH was served as a loading control, and protein expression was quantified by optical density obtained using ImageJ software program. 


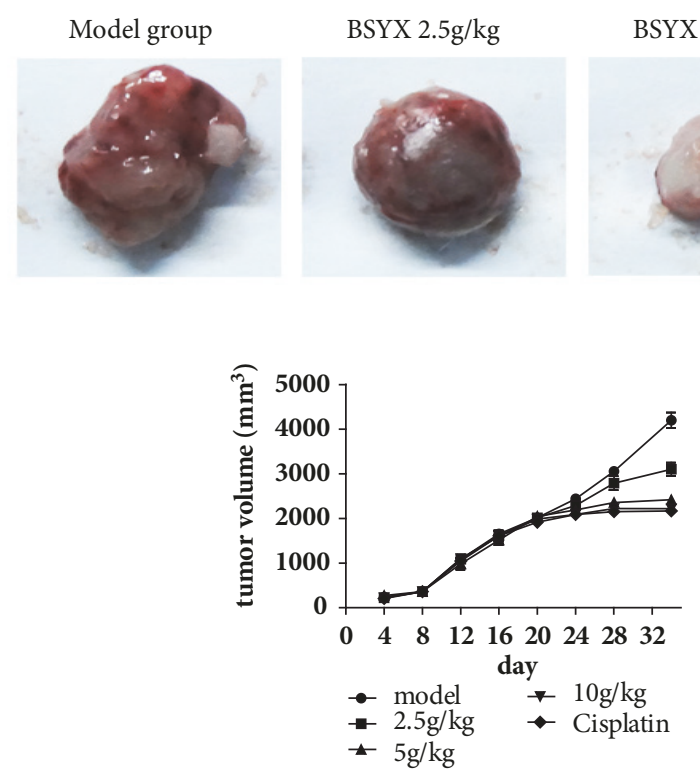

(b)

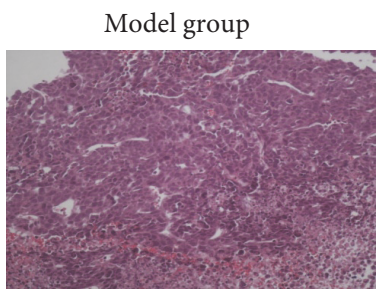

BSYX $5 \mathrm{~g} / \mathrm{kg}$
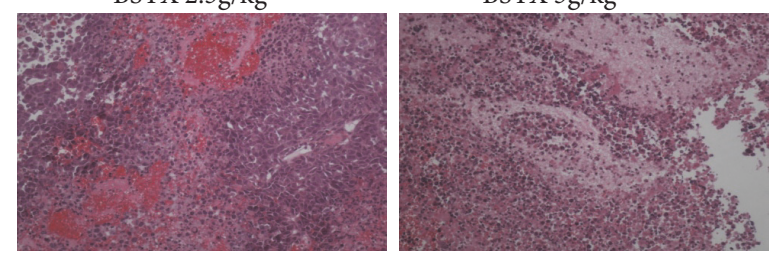

(d)

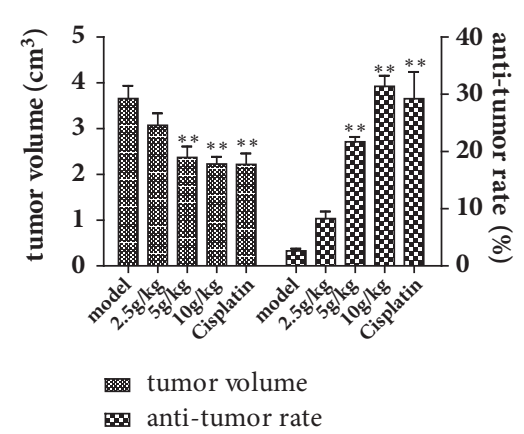

(c)

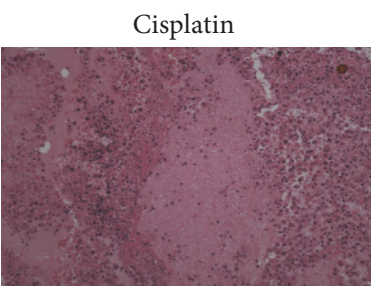

BSYX $10 \mathrm{~g} / \mathrm{kg}$

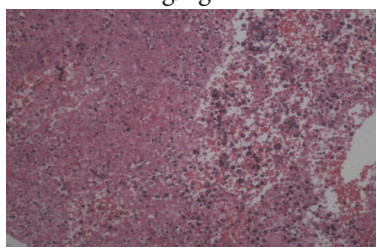

FIGURE 1: Effect of BSYX on Tumor volume and histomorphology in mice tumor issue. After treating for 14 days, tumors from mice were collected (a), growth curve of tumors was obtained from tumor measure every four days (b), and the tumor volume and antitumor rate (c) were calculated. The tumor issue was observed with stained with $\mathrm{H} \& \mathrm{E}$ (magnification of all figures $\times 200)(\mathrm{d}) . * * \mathrm{P}<0.01$ versus the model group.

2.11. Statistical Analysis. The experimental data were analyzed using SPSS 19.0 software. Comparisons among multiple groups were made with one-way analysis of variance followed by Fisher's least significant difference test, and all data were the mean \pm standard deviation of at least three separate experiments; $P<0.05$ was considered statistically significant.

\section{Results}

3.1. Effect of BSYX on Tumor Volume and Histomorphology in Mice. After treatment for 14 days, we collected the tumor and calculated tumor volumes and the anti-tumor rates. As shown in Figures 1(a) and 1(b), the xenograft model of human endometrial carcinoma in nude mice was successfully prepared. The tumor volumes and antitumor rates in the treating groups treated with $\operatorname{BSYX}(5$ and $10 \mathrm{~g} / \mathrm{kg})$ and cisplatin were reduced compared with model group $(\mathrm{P}<0.01)$ (Figure $1(\mathrm{c})$ ). $\mathrm{H} \& \mathrm{E}$ staining was shown in tumor tissue (Figure $1(\mathrm{~d})$ ). The tumor cells in the model group were closely arranged, with lots and different sizes of cancer cells or cell nucleus, less interstitium, and asymmetrical pathological nuclear division phases. In BSYX $2.5 \mathrm{~g} / \mathrm{kg}$ group, tumor cells are dense and irregularly arranged, with large nuclei, deep staining, and numerous mitotic figures. In BSYX 5 and $10 \mathrm{~g} / \mathrm{kg}$ group tumor cells are arranged loosely, with regular morphology and fewer mitotic figures, and the BSYX $10 \mathrm{~g} / \mathrm{kg}$ group was the better. 


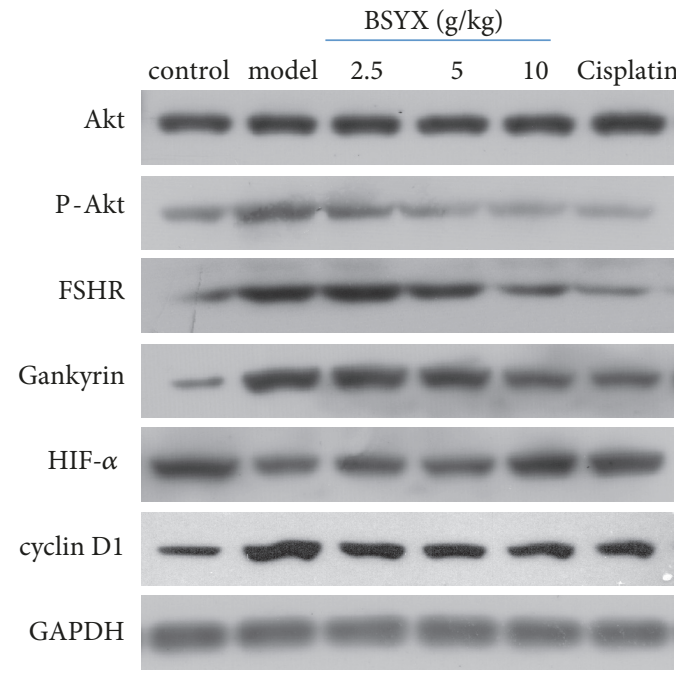

(a)
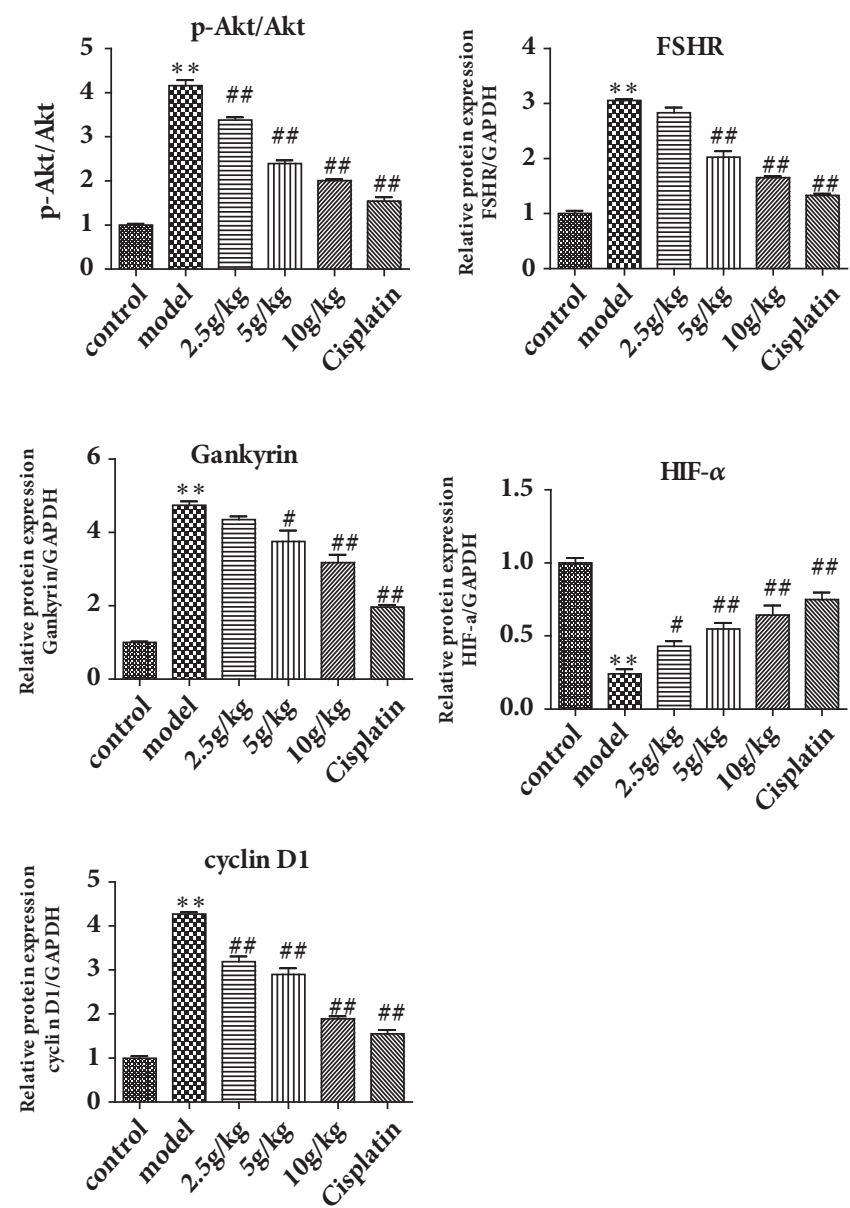

(b)

Figure 2: Effect of BSYX on levels of AKT, p-Akt, FSHR, Gankyrin, HIF-a, and cyclin D1 proteins in mice tumor. AKT, p-AKT, FSHR, Gankyrin, HIF-a, and cyclin D1 proteins levels were measured with Western blotting and normalized to GAPDH (a). Relative band intensities were used in order to quantify AKT, p-Akt, FSHR, Gankyrin, HIF- $\alpha$, and cyclin D1 protein (b) expression levels. Data are expressed as the mean \pm S.E.M. $\mathrm{n}=10$ mice in each group. $* * \mathrm{P}<0.01$ versus the control group, ${ }^{\#} \mathrm{P}<0.05$, and ${ }^{\# \#} \mathrm{P}<0.01$ versus the model group.

3.2. Effect of BSYX on Levels of AKT, p-AKT, FSHR, Gankyrin, HIF- $\alpha$, and Cyclin D1 Proteins in Mice Tumor. The Akt pathway has been frequently reported in cancer, so we examined Akt phosphorylation status. The results showed an evident increase of $\mathrm{p}$-Akt/Akt in model group $(P<0.01)$. Compared with the model group, $\mathrm{p}-\mathrm{Akt} / \mathrm{Akt}$ protein expression was reduced in the treating groups treated with different dose of BSYX and cisplatin $(P<0.01)$. FSHR and Gankyrin/HIF- $\alpha /$ cyclinD1 were upstream and downstream genes of P13K/Akt signal pathway, respectively, then their proteins expression levels were detected. As shown in Figures 2(a) and 2(b), in model group, the levels of FSHR, Gankyrin and cyclinD1 were distinctly increased $(P<0.01)$ and the levels of HIF- $\alpha$ were distinctly decreased $(P<0.01)$. Compared with the model group, the levels of FSHR, Gankyrin, and cyclinD1 in BSYX group were distinctly decreased $(P<0.01)$, and the levels of HIF- $\alpha$ were distinctly increased $(P<0.01)$ in a concentration dependent manner.
3.3. Effect of BSYX on Cell Ability and Apoptosis Rate in Human Endometrial Carcinoma Ishikawa Cells. Human endometrial carcinoma Ishikawa cells were stimulated for $24 \mathrm{~h}$ with serum of BSYX in a gradient concentration, FSH and LY294002, respectively. Cell ability was detected by CCK8 assay. As shown in Figure 3(a), compared with control group, FSH improved the tumor cell viability $(P<0.01)$, and LY294002 reduced the tumor cell viability $(P<0.01)$, cell viability was decreased significantly with increasing BSYX concentrations $(P<0.01)$. Cell death after treatment with BSYX was also measured using flow cytometric analysis with Annexin V-FITC/PI staining. As shown in Figure 3(b), compared with control group, LY294002 promoted the tumor cell apoptosis, which was about $25.78 \pm 1.04 \%(P<0.01)$, cell apoptosis rate was increased significantly in $\operatorname{BSYX}$ group $(P<0.01)$ and was also higher than the LY294002 group. In addition, the proapoptosis protein Bax and the antiapoptosis $\mathrm{BCl}-2$ were observed. As shown in Figure 4, compared with control group, FSH decreased the levels of Bax protein expression 


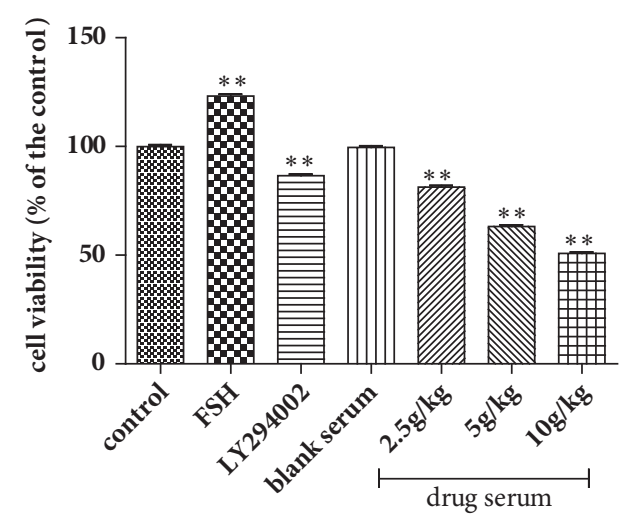

(a)

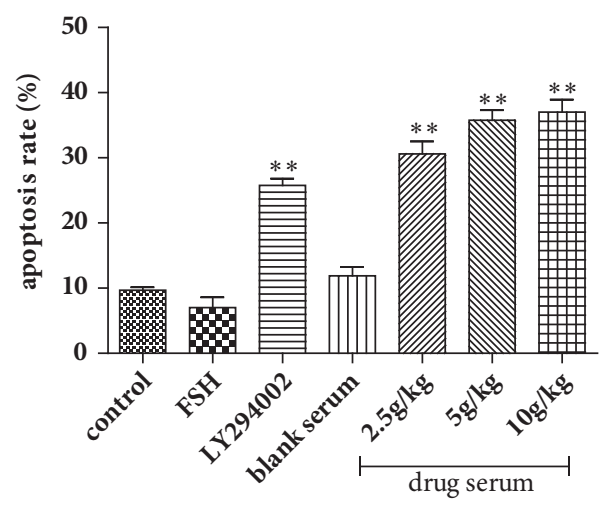

(b)
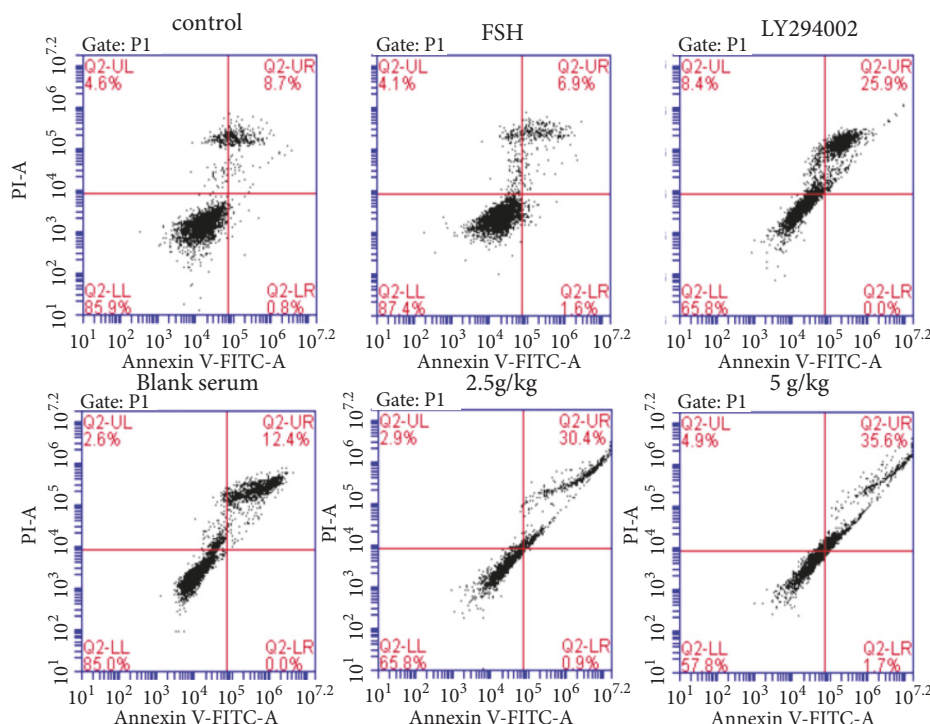

$10 \mathrm{~g} / \mathrm{kg}$

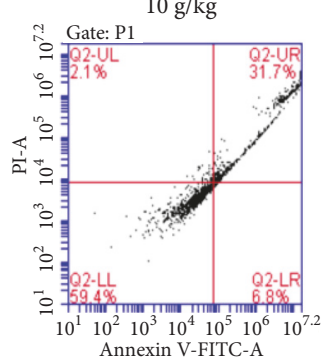

(c)

FIGURE 3: Effect of BSYX on cell ability and apoptosis rate in human endometrial carcinoma Ishikawa cells. Cells were treated with FSH, LY284002, blank serum and drug serum containing BSYX for $24 \mathrm{~h}$, then cell ability was detected by CCK8 assay and was calculated (a). Cells were double stained with annexin V-FITC and PI and analyzed by flow cytometry. And percentage of apoptotic cells was shown by histogram ((b) and (c)). Data are expressed as the mean \pm SEM $n=3$ in each group. $* \mathrm{P}<0.05, * * \mathrm{P}<0.01$ versus the control group.

$(P<0.05)$ and increased the levels of Bcl-2, LY294002 and BSYX increased the levels of Bax protein expression and decreased the levels of $\mathrm{Bcl}-2(\mathrm{P}<0.05, \mathrm{P}<0.01)$,

\subsection{Effect of BSYX on Migration Ability in Human Endome-} trial Carcinoma Ishikawa Cells. Ishikawa cells were stimulated for $24 \mathrm{~h}$ or $48 \mathrm{~h}$ with serum of BSYX in a gradient concentration. As shown in Figure 5, compared with control group, FSH increased the tumor cell migration ability $(P<0.01)$, LY294002 decreased the tumor cell migration ability, which were about $25.62 \pm 0.83 \%$ and $51.29 \pm 0.94 \%$, respectively, at 24 and $48 \mathrm{~h}(P<0.01)$. Cell migration ability in BSYX groups were decreased significantly and lower than those in the control and LY294002 groups $(P<0.01)$.

3.5. FSH/AKT/Gankyrin/HIF- $\alpha /$ CyclinD 1 Pathway Plays an Important Role in BSYX Impact on Human Endometrial Carcinoma Ishikawa Cells. After Ishikawa cells were stimulated for $24 \mathrm{~h}$ with serum of BSYX in a gradient concentration, AKT, p-AKT, FSHR, Gankyrin, HIF-a, and cyclin D1 proteins were detected by western blot analysis. As shown in Figure 6, compare with control group, FSH increased the levels of pAKT/AKT, FSHR, Gankyrin, and cyclinD1 proteins expression $(P<0.05, P<0.01)$ and decreased the levels of HIF- $\alpha$ $(P<0.01)$, LY294002 decreased the levels of $\mathrm{p}-\mathrm{AKT} / \mathrm{AKT}$, FSHR, Gankyrin, and cyclinD1 proteins expression $(\mathrm{P}<0.05$, $\mathrm{P}<0.01)$ and increased the levels of HIF- $\alpha(P<0.01)$, the levels of $\mathrm{p}$-AKT/AKT, FSHR, Gankyrin, and cyclinD1 proteins expression were decreased significantly, and the levels of HIF- $\alpha$ were increased with increasing BSYX concentrations $(P<0.01)$.

\section{Discussion}

The incidence of endometrial cancer is increasing year by year and it is the second leading cause of deaths due to gynaecological cancer worldwide. Recent evidence showed that TCM has great potential in treating endometrial cancer including Qingrejiedu Lianhua soup [17], astragalus polysaccharides [18], and Lithospermum [19]. In our experiment, BSYX prescription, as the classic prescription for treating gynecological diseases in TCM, was chosen to explore its effect on endometrial cancer, Firstly, according to the body 


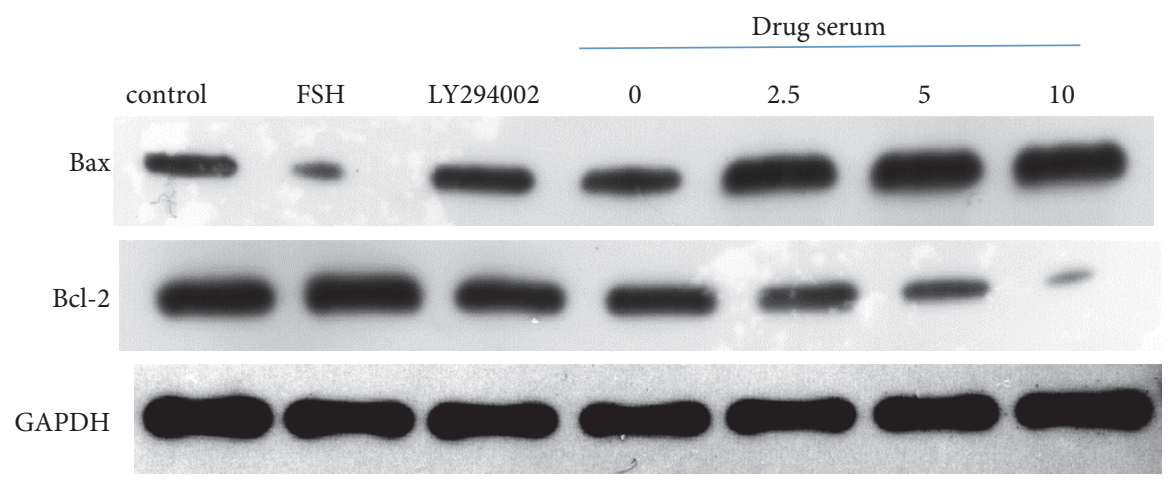

(a)
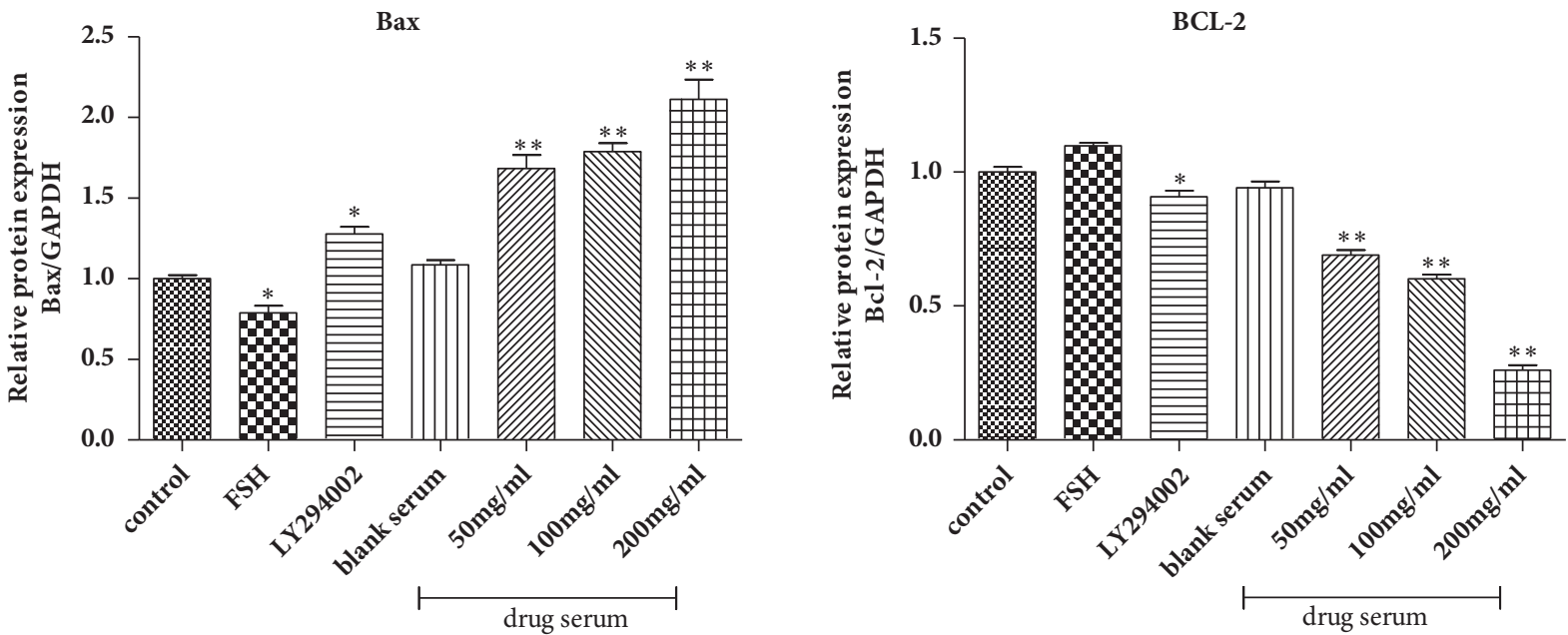

(b)

FIGURE 4: Effect of BSYX on levels of Bax and Bcl-2 proteins in human endometrial carcinoma Ishikawa cells. Bax and Bcl-2 proteins levels were measured with Western blotting and normalized to GAPDH (a). Relative band intensities were used in order to quantify Bax and Bcl-2 proteins (b) expression levels. Data are expressed as the mean $\pm \mathrm{SEM} \mathrm{n}=3$ in each group. $* \mathrm{P}<0.05, * * \mathrm{P}<0.01$ versus the control group.

surface area ratio of mice to human beings, the dosage conversion coefficient for mice to human beings is 9.1, thus, the dose ranges of BSYX in a $60-\mathrm{kg}$ person are equivalent to $3-10 \mathrm{~g} / \mathrm{kg}$. In this study, the doses of $2.5,5$, and $10 \mathrm{~g} / \mathrm{kg}$ for 14 days were used in the treatment of the mice. The models of subcutaneous tumor xenograft of nude mice were established by injection of human endometrial carcinoma cell line Ishikawa tumor cell suspension. Our results indicated that the tumor volume in 5 and $10 \mathrm{~g} / \mathrm{kg}$ BSYX group was lower while the inhibition rate was higher than those in the model group, consistent with the change in pathological morphology.

The endometrium is now considered to be two kinds of pathogenesis: one is a kind of estrogen dependent; namely, endometrial hyperplasia or even cancer may occur under the long-term action of estrogen without progesterone antagonism. This type accounts for the majority of endometrial cancer. The other is nonestrogen-dependent and has no definite relationship with estrogen [20]. The effect of FSH on target organs is mainly mediated by receptor FSHR. FSHR is not only expressed in female ovarian granule cells and male testicular cells, but also in prostate [21], ovarian epithelium
[8], and other tissues. Aberrant FSHR function facilitates the development of cancer, and FSH promotes proliferation of ovarian cells [14]. PI3K/Akt pathway can be regulated by FSHR, and PI3K/Akt pathway activation was associated with enhanced invasive and migratory capacities. Inhibiting the PI3K/Akt pathway reversed progestin resistance in endometrial cancer [22]. Meanwhile, Gankyrin is crucial for breast cancer metastasis and highlight the potential of Gankyrin as a therapeutic target for tumor metastasis [23]. What is more, high expression level of Gankyrin increased hepatocellular carcinoma cell migration and invasion by PI3K/Akt pathway [24]. In addition, Gankyrin could induce cyclin D1 expression [25]. Cyclins play key roles in cell proliferation and growth. Cyclin D1 is an important mediator of cancer initiation, development, and metastasis and is associated with poor prognosis. LncRNA ABHD11-AS1 promotes the development of endometrial carcinoma by targeting cyclin D1[26]. Interfering with cyclin D1 leads to PI3K/Akt signal suppression [27]. So the signal transduction pathway of FSH, PI3K/Akt, and Gankyrin/HIF- $\alpha /$ cyclinD1 in endometrial carcinoma attracted our interest. Our results found that BSYX induced an increase in the expression levels of FSHR, Akt/p-Akt, 


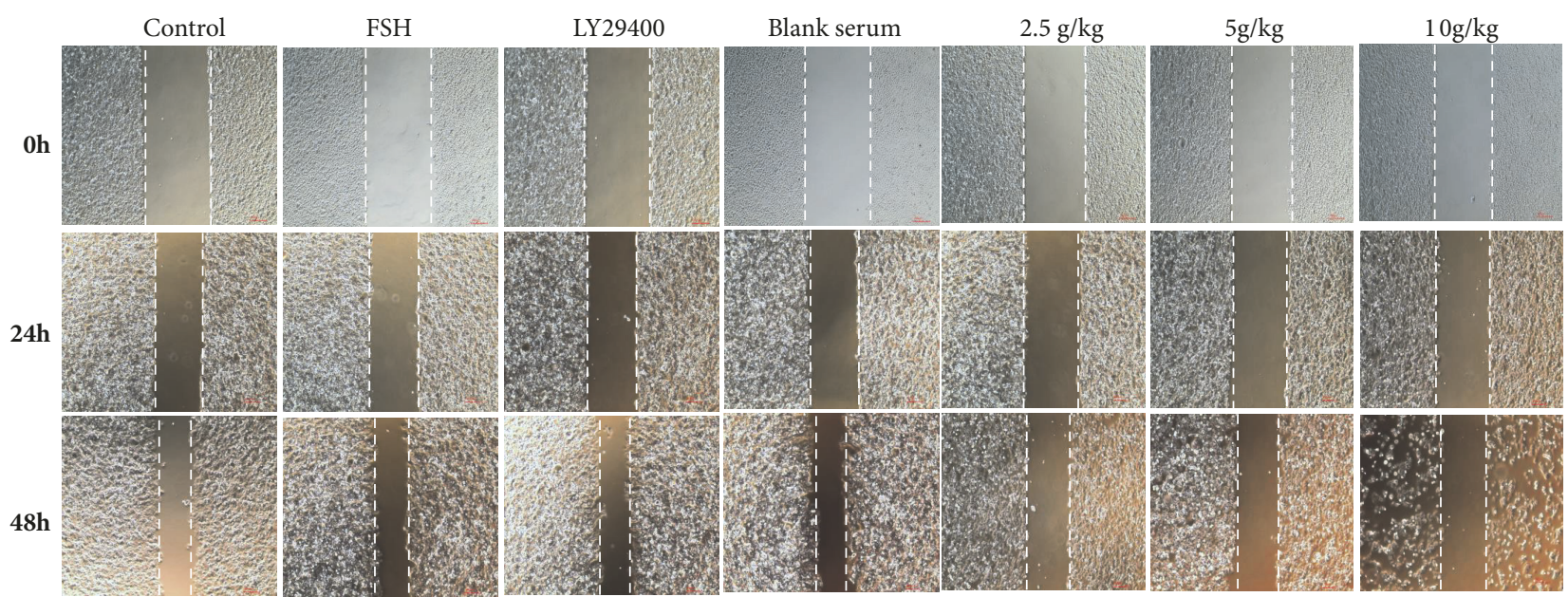

(a)

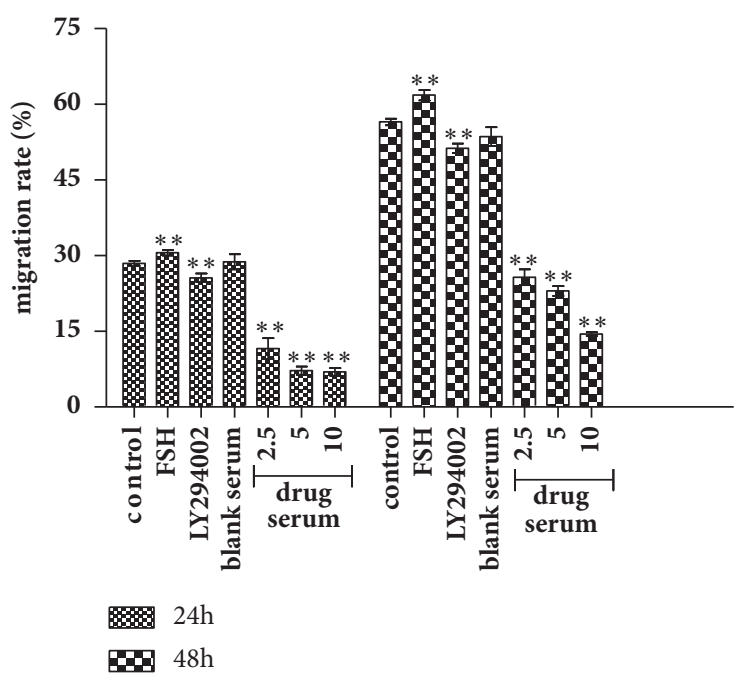

(b)

FIGURE 5: Effect of BSYX on migration ability in human endometrial carcinoma Ishikawa cells After BSYX treatment, the cell migration ability was analyzed by the wound healing assays (a) and the migration rate was calculated (b). Data are expressed as the mean \pm SEM $n=3$ in each group. $* \mathrm{P}<0.05, * * \mathrm{P}<0.01$ versus the control group.

Gankyrin, and cyclin D1 proteins and a decrease in HIF- $\alpha$ in mice tumor. Therefore the relationship of endometrial carcinoma and the FSH/PI3K/AKT/Gankyrin/HIF- $\alpha$ /cyclinD1 pathway was further discussed with BSYX intervention in vitro.

LY294002, a highly selective PI3K inhibitor, was used to block PI3K-dependent Akt phosphorylation. When exposed Ishikawa cells to FSH and LY294002, LY294002 reduced the tumor cell viability, increased cell apoptosis rate and proapoptosis Bax protein expression, decreased the anti-apoptosis $\mathrm{Bcl}-2$ protein expression and the tumor cell migration ability, downregulated the levels of p-AKT/Akt, FSHR, Gankyrin, and cyclinD1 proteins expression, and upregulated the levels of HIF- $\alpha$. However, FSH increased the levels of FSHR, pAKT/Akt, Gankyrin, and cyclinD1 proteins expression and decreased the levels of HIF- $\alpha$. The results obtained from exposed Ishikawa cells to BSYX were in line with LY294002.
BSYX led a decrease in the tumor cell viability and cell migration ability and changed the expression levels of $\mathrm{p}$ AKT/Akt, FSHR, Gankyrin and cyclinD1 and proteins HIF$\alpha$ which were in line with those in vivo. In the next step, we plan to explore the specific components of BSYX prescription in combination with antiendometrial cancer and the mechanism of action through network pharmacology in order to provide powerful new knowledge to guide its clinical usage.

\section{Conclusions}

To sum up, 5 and $10 \mathrm{~g} / \mathrm{kg}$ BSYX reduced tumor volume, changed pathological feature in mice tumor issue, also markedly reduced the human endometrial carcinoma Ishikawa cell growth ability and migration ability, and accelerated the apoptosis. The formulation of BSYX had 


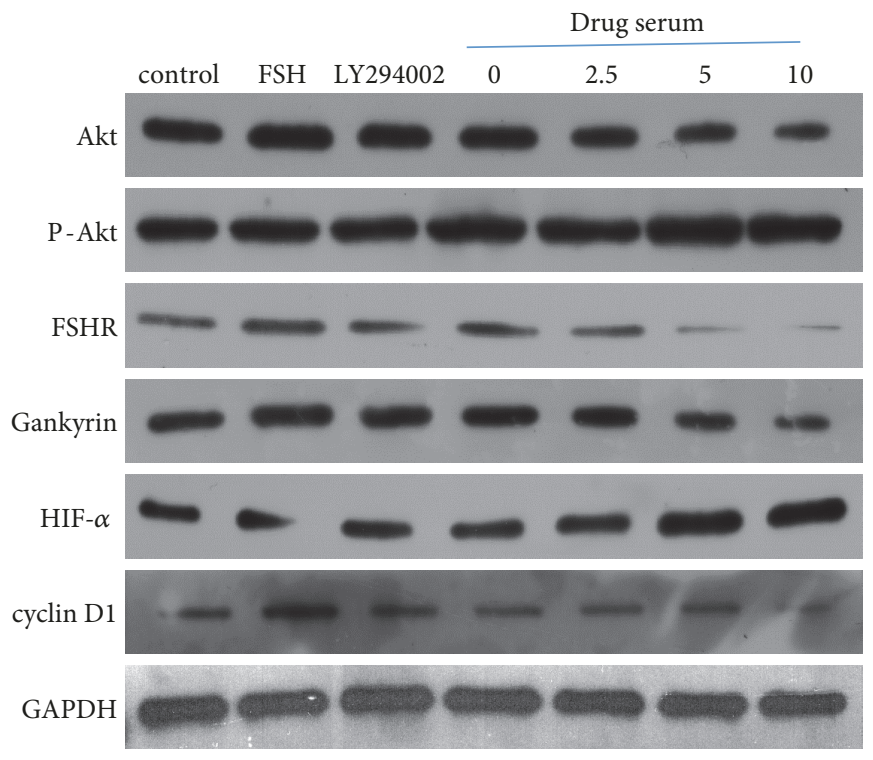

(a)
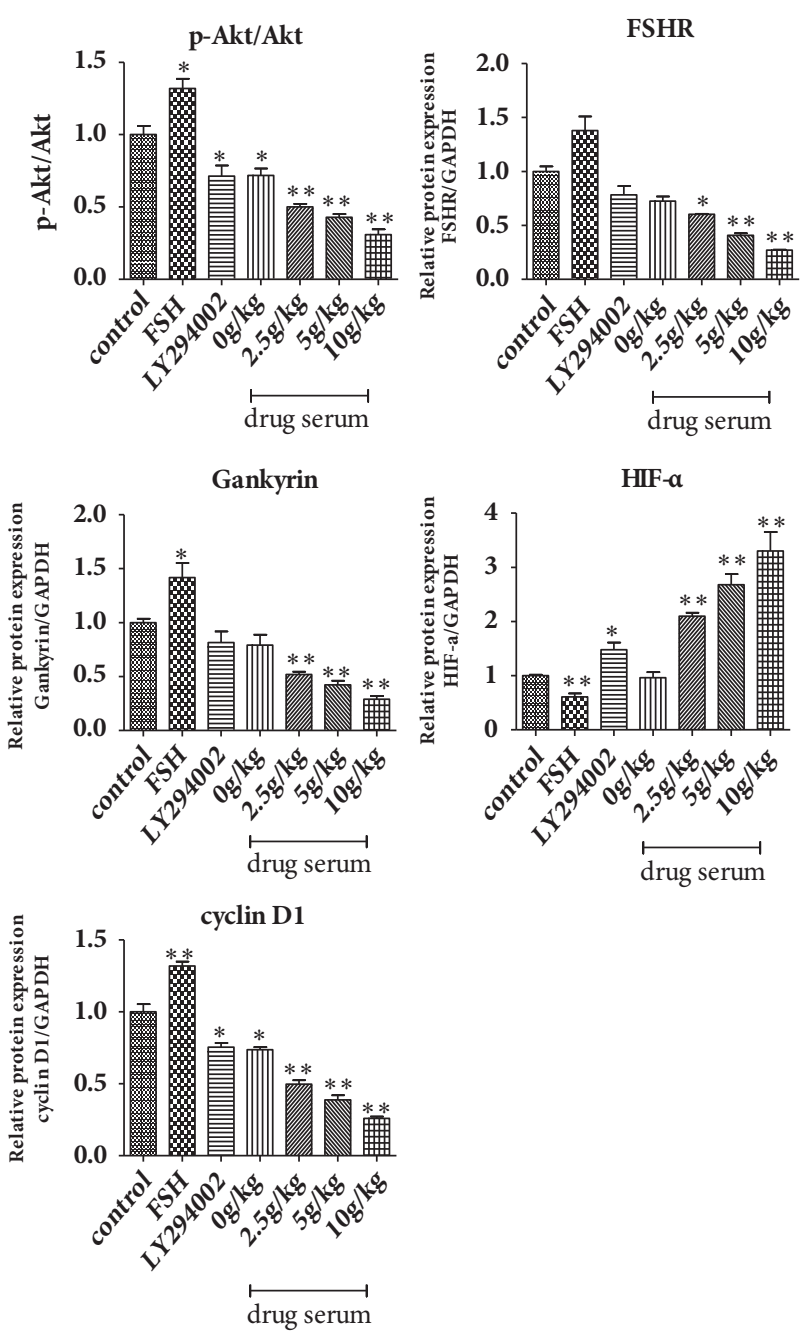

(b)

FIGURE 6: Effect of BSYX on levels of AKT, p-Akt, FSHR, Gankyrin, HIF-a, and cyclin D1 proteins in human endometrial carcinoma Ishikawa cells. AKT, p-Akt, FSHR, Gankyrin, HIF- $\alpha$, and cyclin D1 proteins levels were measured with Western blotting and normalized to GAPDH(a). Relative band intensities were used in order to quantify AKT, p-AKT, FSHR, Gankyrin, HIF- $\alpha$, and cyclin D1 protein (b) expression levels. Data are expressed as the mean \pm SEM $\mathrm{n}=3$ in each group. $* \mathrm{P}<0.05, * * \mathrm{P}<0.01$ versus the control group.

treating effect on endometrial cancer and was related with FSH/PI3K/AKT/Gankyrin/HIF- $\alpha /$ cyclinD1 pathway, which can supply the experimental basement of its clinical usage.

\section{Data Availability}

The data used to support the findings of this study are included within the article.

\section{Conflicts of Interest}

The authors declare that they have no conflicts of interest regarding the publication of this article.

\section{Authors' Contributions}

Yue-qun Chen carried out the conception and design of the research, participated in the acquisition of data, and drafted the manuscript. Hua-li Fei carried out the analysis and interpretation of data. Hong-li Zhu conceived of the study and participated in its design and coordination and helped to draft the manuscript. All authors read and approved the final manuscript.

\section{References}

[1] A. Kim, L. Nguyen, T. Kalir, and L. Chuang, "Pelvic recurrence of stage la well-differentiated endometrial carcinoma after 13 years: A case report," Journal of the Turkish German Gynecological Association, vol. 17, no. 1, pp. 51-54, 2016.

[2] Y. Liao, W. Lu, Q. Che et al., "SHARP1 suppresses angiogenesis of endometrial cancer by decreasing hypoxia-inducible factor$1 \alpha$ level," PLoS ONE, vol. 9, no. 6, p. e99907, 2014.

[3] L. A. Torre, F. Bray, R. L. Siegel, J. Ferlay, and J. LortetTieulent, "Global cancer statistics, 2012," CA: A Cancer Journal for Clinicians, vol. 65, no. 2, pp. 87-108, 2015. 
[4] N. Bansal, V. Yendluri, and R. M. Wenham, "The molecular biology of endometrial cancers and the implications for pathogenesis, classification, and targeted therapies," Cancer Control, vol. 16, no. 1, pp. 8-13, 2009.

[5] L. Cai, H. Li, and Y. Wei, "Effect of Yangxue bushen tablet on ovarian function in animal model of Yang deficiency," Chinese Journal of Integrative Medicine, vol. 18, no. 10, pp. 620-622, 1998.

[6] J. Zhang and X. Wang, "Influence of BUSHEN YANGXUE Recipe on Cervical Mucus and Endometrium in Clomiphene Citrate Cycle," Journal of Fujian College of TCM, vol. 13, no. 04, pp. 6-8, 2003.

[7] X. Dong, S. Liu, D. Lii, J. Zhou, and N. Ye, "Effects of Bushen Yangxue Prescription on Expression of Bcl-2 and Bax Protein in Mice Ovaries with Immune Premature Ovarian Failure," Chinese Journal of Experimental Traditional Medical Formulae, vol. 20, no. 1, pp. 134-138, 2014.

[8] A. La Marca, A. C. Artenisio, G. Stabile, F. Rivasi, and A. Volpe, "Evidence for cycle-dependent expression of folliclestimulating hormone receptor in human endometrium," Gynecological Endocrinology, vol. 21, no. 6, pp. 303-306, 2005.

[9] H. Bai, H. Li, W. Li et al., "The PI3K/AKT/mTOR pathway is a potential predictor of distinct invasive and migratory capacities in human ovarian cancer cell lines," Oncotarget , vol. 6, no. 28, pp. 25520-25532, 2015.

[10] K.-X. Sun, D.-D. Wu, S. Chen, Y. Zhao, and Z.-H. Zong, "LncRNA MEG3 inhibit endometrial carcinoma tumorigenesis and progression through PI3K pathway," Apoptosis, vol. 22, no. 12, pp. 1543-1552, 2017.

[11] L. W. Cheung, B. T. Hennessy, J. Li et al., "High frequency of PIK3R1 and PIK3R2 mutations in endometrial cancer elucidates a novel mechanism for regulation of PTEN protein stability," Cancer Discovery, vol. 1, no. 2, pp. 170-185, 2011.

[12] C. He and D. J. Klionsky, "Regulation mechanisms and signaling pathways of autophagy," Annual Review of Genetics, vol. 43, pp. 67-93, 2009.

[13] J. Zhang, Y. Yang, Z. Zhang et al., "Gankyrin plays an essential role in estrogen-driven and GPR30-mediated endometrial carcinoma cell proliferation via the PTEN/PI3K/AKT signaling pathway," Cancer Letters, vol. 339, no. 2, pp. 279-287, 2013.

[14] J. Chen, M. Bai, C. Ning et al., "Gankyrin facilitates folliclestimulating hormone-driven ovarian cancer cell proliferation through the PI3K/AKT/HIF-1 $\alpha /$ cyclin D1 pathway," Oncogene, vol. 35, no. 19, pp. 2506-2517, 2016.

[15] S. Davies, C. M. R. Bax, E. Chatzaki, T. Chard, and R. K. Iles, "Regulation of endometrial cancer cell growth by luteinizing hormone (LH) and follicle stimulating hormone (FSH)," British Journal of Cancer, vol. 83, no. 12, pp. 1730-1734, 2000.

[16] R. Guo, L. Wei, Z. Tu et al., "17 $\beta$-Estradiol activates PI3K/Akt signaling pathway by estrogen receptor (ER)-dependent and ER-independent mechanisms in endometrial cancer cells," The Journal of Steroid Biochemistry and Molecular Biology, vol. 99, no. 1, pp. 9-18, 2006.

[17] T. Y. Bao, L. Zn, and X. Xo, "Effect and mechanism of Qingrejiedu Lianhua soup extracted on autophagy of HEC-1A and Ishikawa cells in endometrial carcinoma," Chinese Journal of Clinical Obstetrics and Gynecology, vol. 19, no. 2, pp. 150-154, 2018.

[18] QIU. Yanli, Yan. DING, and C. Desen, "Effect of astragalus polysaccharides on WNT transduction pathway in subcutaneous tumor xenograft in nude mice with human endometrial carcinoma," Modern Journal of Integrated Traditional Chinese and Western Medicine, vol. 27, no. 11, pp. 1145-1148, 2018.
[19] W. Xie and X. Xue, "Effects of Shikonin on Proliferation and Apoptosis Signal Pathway PI3K / PKB in Vitro Endometrial Carcinoma Cell Line, ACTA CHINESE MEDICINE," Effects of Shikonin on Proliferation and Apoptosis Signal Pathway PI3K / $P K B$ in Vitro Endometrial Carcinoma Cell Line, ACTA CHINESE MEDICINE, vol. 32, no. 12, pp. 2280-2283, 2017.

[20] N. G. Ordóñez, "Value of thyroid transcription factor-1 immunostaining in tumor diagnosis: A review and update," Applied Immunohistochemistry \& Molecular Morphology, vol. 20, no. 5, pp. 429-444, 2012.

[21] S. Mariani, L. Salvatori, S. Basciani et al., "Expression and Cellular Localization of Follicle-Stimulating Hormone Receptor in Normal Human Prostate, Benign Prostatic Hyperplasia and Prostate Cancer," The Journal of Urology, vol. 175, no. 6, pp. 2072-2077, 2006.

[22] C. Gu, Z. Zhang, Y. Yu et al., "Inhibiting the PI3K/Akt pathway reversed progestin resistance in endometrial cancer," Cancer Science, vol. 102, no. 3, pp. 557-564, 2011.

[23] C. Zhen, L. Chen, Q. Zhao et al., "Gankyrin promotes breast cancer cell metastasis by regulating Racl activity," Oncogene, vol. 32, no. 29, pp. 3452-3460, 2013.

[24] W. Sun, J. Ding, K. Wu et al., "Gankyrin-mediated dedifferentiation facilitates the tumorigenicity of rat hepatocytes and hepatoma cells," Hepatology, vol. 54, no. 4, pp. 1259-1272, 2011.

[25] Z. Bai, Y. Tai, W. Li et al., "Gankyrin activates IL-8 to promote hepatic metastasis of colorectal cancer," Cancer Research, vol. 73, no. 14, pp. 4548-4558, 2013.

[26] Y. Liu, L. Wang, S. Chen, Z. Zong, X. Guan, and Y. Zhao, "LncRNA ABHD11-AS1 promotes the development of endometrial carcinoma by targeting cyclin D1," Journal of Cellular and Molecular Medicine, vol. 22, no. 8, pp. 3955-3964, 2018.

[27] C. Chen, Y. Wang, S. Wang et al., "LSD1 sustains estrogendriven endometrial carcinoma cell proliferation through the PI3K/AKT pathway via di-demethylating H3K9 of cyclin D1," International Journal of Oncology, vol. 50, no. 3, pp. 942-952, 2017. 


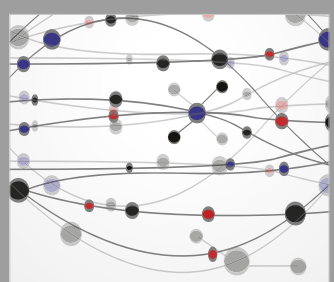

The Scientific World Journal
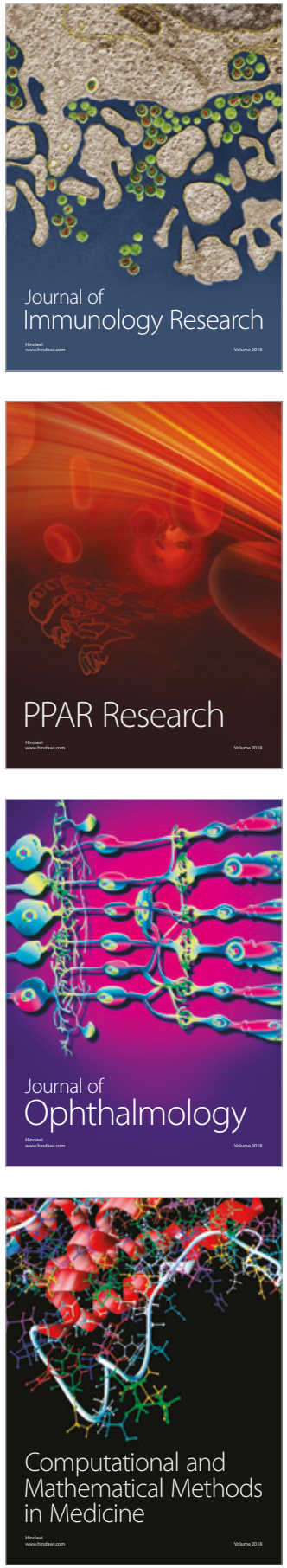

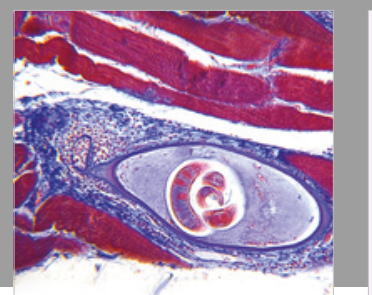

Gastroenterology Research and Practice

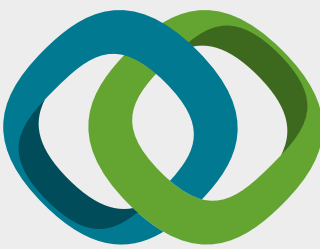

\section{Hindawi}

Submit your manuscripts at

www.hindawi.com
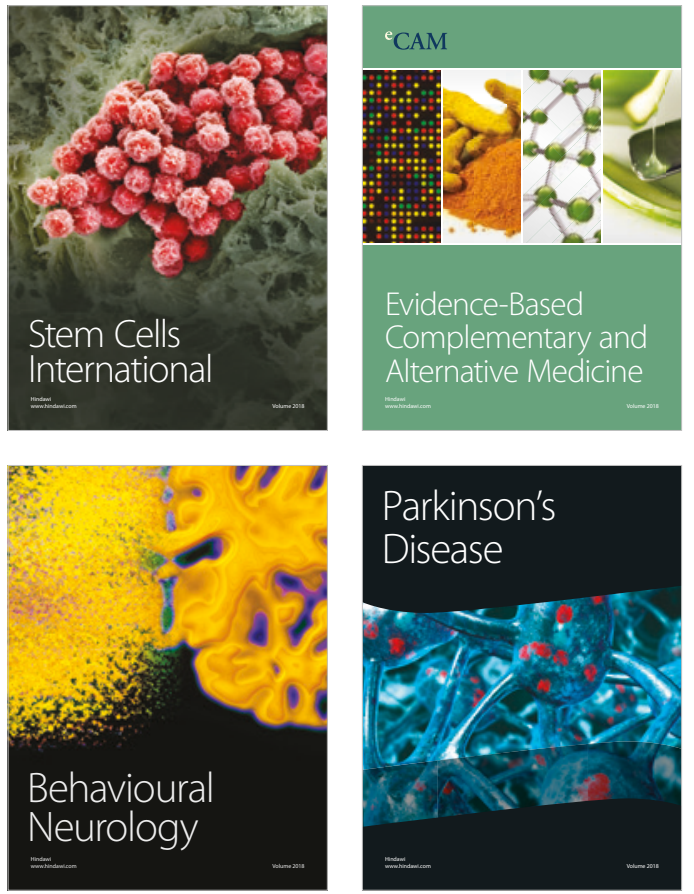

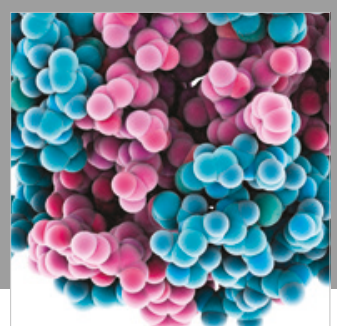

ournal of

Diabetes Research

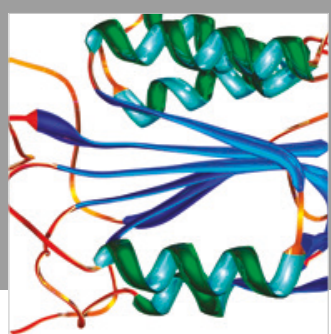

Disease Markers
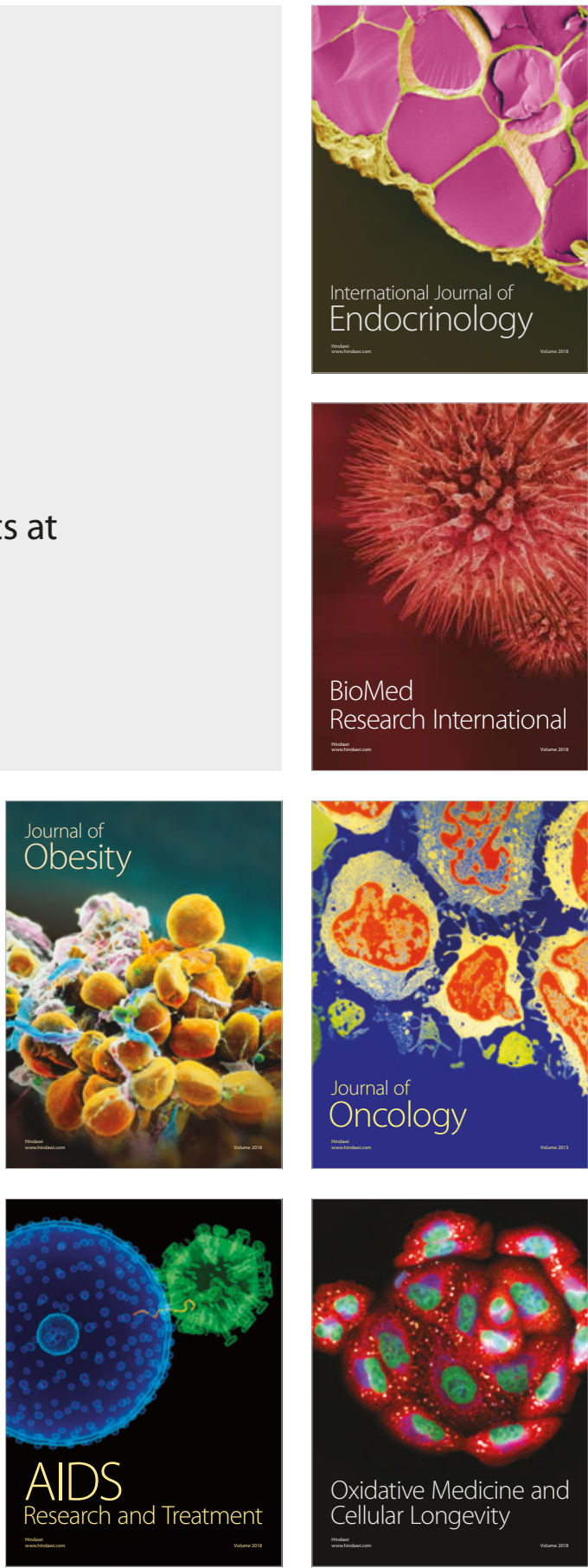